\title{
Long-Acting Intranasal Insulin Detemir Improves Cognition for Adults with Mild Cognitive Impairment or Early-Stage Alzheimer's Disease Dementia
}

\author{
Amy Claxton $^{\mathrm{a}, \mathrm{b}}$, Laura D. Baker ${ }^{\mathrm{c}}$, Angela Hanson ${ }^{\mathrm{a}, \mathrm{b}}$, Emily H. Trittschuh ${ }^{\mathrm{a}, \mathrm{b}}$, Brenna Cholerton ${ }^{\mathrm{b}}$, \\ Amy Morgan $^{\mathrm{a}, \mathrm{b}}$, Maureen Callaghan ${ }^{\mathrm{a}, \mathrm{b}}$, Matthew Arbuckle ${ }^{\mathrm{d}}$, Colin Behl ${ }^{\mathrm{a}, \mathrm{b}}$ and Suzanne Craft ${ }^{\mathrm{c}, *}$ \\ ${ }^{a}$ Geriatric Research, Education, \& Clinical Center, Veterans Affairs Puget Sound Health Care System, Seattle, \\ Washington, USA \\ ${ }^{\mathrm{b}}$ Department of Psychiatry \& Behavioral Science, University of Washington School of Medicine, Seattle, \\ Washington, USA \\ ${ }^{\mathrm{c}}$ Department of Internal Medicine, Wake Forest School of Medicine, Winston-Salem, North Carolina, USA \\ ${ }^{\mathrm{d}}$ Department of Psychiatry, Oregon Health and Science University, Portland, Oregon, USA
}

Accepted 29 September 2014

\begin{abstract}
Previous trials have shown promising effects of intranasally administered insulin for adults with Alzheimer's disease dementia (AD) or amnestic mild cognitive impairment (MCI). These trials used regular insulin, which has a shorter half-life compared to long-lasting insulin analogues such as insulin detemir. The current trial examined whether intranasal insulin detemir improves cognition or daily functioning for adults with MCI or AD. Sixty adults diagnosed with MCI or mild to moderate AD received placebo $(n=20), 20$ IU of insulin detemir $(n=21)$, or 40 IU of insulin detemir $(n=19)$ for 21 days, administered with a nasal drug delivery device. Results revealed a treatment effect for the memory composite for the 40 IU group compared with placebo $(p<0.05)$. This effect was moderated by APOE status $(p<0.05)$, reflecting improvement for APOE- $\varepsilon 4$ carriers $(p<0.02)$, and worsening for non-carriers $(p<0.02)$. Higher insulin resistance at baseline predicted greater improvement with the $40 \mathrm{IU}$ dose $(r=0.54, p<0.02)$. Significant treatment effects were also apparent for verbal working memory $(p<0.03)$ and visuospatial working memory $(p<0.04)$, reflecting improvement for subjects who received the high dose of intranasal insulin detemir. No significant differences were found for daily functioning or executive functioning. In conclusion, daily treatment with $40 \mathrm{IU}$ insulin detemir modulated cognition for adults with AD or MCI, with APOE-related differences in treatment response for the primary memory composite. Future research is needed to examine the mechanistic basis of APOE-related treatment differences, and to further assess the efficacy and safety of intranasal insulin detemir.
\end{abstract}

Keywords: Alzheimer's disease, clinical trials, randomized, insulin, intranasal drug administration, mild cognitive impairment

\section{INTRODUCTION}

The importance of insulin in normal brain function is underscored by evidence that insulin dysregulation

*Correspondence to: Suzanne Craft, PhD, Wake Forest School of Medicine, Medical Center Boulevard, Winston-Salem, NC 271571207, USA. Tel.: +1 336713 8832; Fax: +1 336713 8800; E-mail: suzcraft@wakehealth.edu. contributes to the pathophysiology of Alzheimer's disease (AD) [1-3]. Research in animals and humans has shown that AD pathology is associated with lower levels of insulin in the cerebrospinal fluid [4]. Insulin has a close relationship with amyloid- $\beta(\mathrm{A} \beta)$, the peptide produced by cleavage of the amyloid- $\beta$ protein precursor. $A \beta$ pathologically aggregates to form plaques in $\mathrm{AD}$, and its oligomeric form is synaptotoxic even 
prior to plaque deposition [5]. In vitro and in animal models, insulin reduces $A \beta$ oligomer formation and protects against $A \beta$-induced synaptotoxicty and long-term potentiation disruption $[2,6]$. Consequently, disruptions in brain insulin signaling have been suggested as one of the primary pathophysiological factors in the development of AD. Clinical studies have documented substantial, progressive disturbances in brain glucose utilization and responsiveness to insulin and insulin-like growth factor stimulation that co-occur with progression of $\mathrm{AD}[7,8]$. Disruption in central insulin regulation (also referred to as brain insulin resistance) induces pathological features of $\mathrm{AD}$, and can be caused by attenuated expression of insulin receptors and insulin-like growth factor, reduced brain insulin receptor sensitivity, or increased serine phosphorylation of downstream insulin signaling molecules $[1,9,10]$. Impaired transport of insulin across the blood-brain barrier may also result in deficient levels of insulin in the central nervous system (CNS). Thus, enhancing brain insulin may prevent AD-related pathological processes.

Recent clinical trials have yielded promising effects of intranasally administered insulin for adults diagnosed with mild AD dementia or amnestic mild cognitive impairment (MCI) [11-13]. In a pilot trial, adults diagnosed with mild $\mathrm{AD}$ or MCI who received a 20 IU daily dose of intranasal insulin improved in delayed memory, and participants receiving either a 20 or $40 \mathrm{IU}$ dose improved on caregiver-rated functional ability [13]. Both doses of insulin were associated with preserved cognition for younger participants on the Alzheimer's Disease Assessment Scale-Cognitive subscale, and preserved glucose uptake was noted for participants taking intranasal insulin on FDG-PET scans in the parietotemporal, frontal, precuneus, and cuneus regions. The improvements in episodic memory were still present two months after cessation of treatment [13].

Prior studies have also demonstrated that treatment response to many $\mathrm{AD}$ therapies is moderated by carriage of the apolipoprotein E- $\varepsilon 4$ (APOE- $\varepsilon 4)$ allele [14]. In particular, research has indicated that both peripheral and central insulin metabolism, as well as insulin-altering therapies, are modulated by the APOE$\varepsilon 4$ allele [4, 15-17]. The APOE- $\varepsilon 4$ allele is known to increase the risk of developing sporadic $\mathrm{AD}$, but the mechanism of action is not fully elucidated [14]. Previous studies have demonstrated APOE- $\varepsilon 4$-related differences in response to various $\mathrm{AD}$ therapies, and in particular to the therapeutic effects of insulin $[16,18]$. In two pilot studies, treatment response was strongest for APOE- $\varepsilon 4$ negative older adults with mild memory problems or $\mathrm{AD}[11,19]$, whereas in another study, APOE- $\varepsilon 4$ positive adults showed greater sensitivity to insulin at lower doses [20]. Other studies have also found interactions between APOE- $\varepsilon 4$ carriage and central insulin or glucose action [18, 21].

The previous clinical trials for AD described above have all used regular insulin, which has a relatively short half-life and mimics post prandial release and in general have observed the most reliable benefits with doses of 20 or $40 \mathrm{IU}$ daily. The long-acting insulin analog insulin detemir, because of the acylation of a 14-carbon fatty acid to lysine at locus B29, displays increased self-association and reversible albumin binding [22], which delays absorption of the molecule and thereby reduces the risk of hypoglycemic episodes [23]). Due to its increased lipophilicity, detemir may reach higher concentrations in the cerebrospinal fluid and brain than regular insulin [24]. The issue of whether detemir crosses the blood-brain barrier (BBB) is controversial. Although it has been described to have greater capacity to cross the BBB with potential for correspondingly increased cognitive benefit [25], elegant work in rodents has suggested that detemir does not penetrate the BBB [26], and thus intranasal or intracerebroventricular administration may be needed to ensure delivery to the brain. Although this issue remains unresolved in humans or in diseases with impaired BBB permeability, detemir has been shown to be as effective or more so than regular insulin at reducing hyperglycemia and nocturnal hypoglycemic episodes [27]. In vivo studies have shown that detemir demonstrates increased insulin-signaling in the hypothalamus and cerebrocortical tissue compared to regular insulin [28], suggesting that detemir may have greater central action compared to regular insulin. Another study comparing euglycemic infusion of detemir with regular insulin reported that detemir triggered a larger shift in EEG DC-potential recordings in healthy men, supporting the hypothesis that detemir affects brain functions to a greater extent than does regular insulin [29]. The peripheral effects of detemir also appear to vary according to baseline metabolic status, with greater effects on weight and other metabolic outcomes observed for adults who are more insulin resistant or obese $[25,30]$.

Intranasal administration of detemir has not previously been tested in adults with neurodegenerative disease. Because it has a different structure than regular insulin, with more prolonged elevations and greater CNS penetration, its safety and efficacy may differ from regular insulin. In the current pilot study, we 
examined the safety profile and efficacy of two doses of insulin detemir for treatment of adults diagnosed with AD or amnestic MCI compared with placebo, using a three week protocol that effectively revealed cognitive and safety profiles in early studies of regular insulin [12], and that would provide necessary information to design future longer trials. We hypothesized that, compared with placebo, intranasal detemir would be associated with improved performance on an episodic memory composite. Secondary hypotheses predicted that daily function and working memory would improve for adults with MCI or AD taking intranasal insulin detemir versus placebo. Given previous findings of APOE-related differences and metabolism-related differences in treatment response, APOE- $\varepsilon 4$ carrier and peripheral metabolic status were examined as possible mediating factors in a priori secondary analyses.

\section{MATERIALS AND METHODS}

\section{Participants}

The trial was registered at clinicaltrials.gov (NCT01547169) and conducted over a 2-year period. The study was approved by the Institutional Review Boards of the University of Washington and the Veterans Affairs Puget Sound Health Care System and was conducted in the Veterans Affairs Clinical Research Unit. Written informed consent was obtained from all participants. A total of 60 older adults enrolled in our study (39 participants with amnestic MCI and 21 participants with probable AD with Mini-Mental State Examination (MMSE) scores $>15$ ). Diagnoses and eligibility were determined by consensus of expert physicians and neuropsychologists following cognitive testing, evaluation of medical history, physical examination, and clinical laboratory screening using modified Petersen criteria for the diagnosis of amnestic MCI [12, 31] and National Institute for Neurological and Communicative Disorders and Stroke-Alzheimer's Disease and Related Disorders Association criteria for AD [32]. For participants with amnestic MCI, cognitive scores were compared with an age- and education-adjusted estimate of the participant's premorbid ability (Shipley Vocabulary test). Participants whose delayed memory scores deviated at least 1.5 SDs from this estimate were considered for the diagnosis of amnestic MCI, which was then determined by expert consensus using all available data, following published criteria [31].
Participants were free from psychiatric disorders, alcoholism, severe head trauma, hypoxia, neurologic disorders other than amnestic MCI or AD, renal or hepatic disease, diabetes mellitus, chronic obstructive pulmonary disease, and unstable cardiac disease. Participants and all study personnel involved in data collection were blinded to treatment assignment. Treatment groups did not differ significantly in terms of age, education, body mass index, general cognitive status as assessed by the modified iMMSE, gender, diagnosis, whether they received anticholinesterase inhibitors or memantine, or whether they carried the APOE$\varepsilon 4$ allele. Enrollment data are presented in Fig. 1, and baseline demographic information is presented in Table 1.

\section{Procedures}

Participants were randomly assigned to receive a daily dosage of $20 \mathrm{IU}$ of insulin detemir (10 IU detemir b.i.d.), $40 \mathrm{IU}$ of insulin detemir (20 IU detemir b.i.d.), or placebo (saline b.i.d.) for 21 days. Saline or insulin detemir (Levemir ${ }^{\circledR}$; Novo Nordisk, Princeton, New Jersey) was administered after breakfast and dinner with a ViaNase nasal drug delivery device (Kurve Technology, Bothell, Washington) designed to deliver drugs to the olfactory cleft region to maximize transport to the CNS. This device released a metered dose of saline or detemir into a chamber covering the participant's nose over a 2-min period, which was inhaled by breathing regularly. The choice of the 20 and 40 IU doses was based on our prior work in which similar doses of regular insulin enhanced cognition [11-13]. Dosing of insulin analogues has been calibrated to regular insulin by the pharmaceutical industry such that IU measurements of regular insulin and insulin detemir are equivalent. As this pilot study represents the first attempt to administer detemir to older adults with neurodegenerative disease, we used 20 and 40 IU doses and a three-week treatment duration that in previous studies of regular insulin provided initial indications of safety and efficacy to support additional longer-term investigation.

Parallel versions of the cognitive and functional protocol were administered at baseline and after 21 days of treatment. Testing occurred in the morning after a standard meal. Participants were instructed to skip their morning dose on the day of testing and thus had received their last dose more than $12 \mathrm{~h}$ prior to cognitive testing. The primary outcome measure was a verbal memory composite score calculated from the sum of $\mathrm{z}$-scores from the following four measures: 


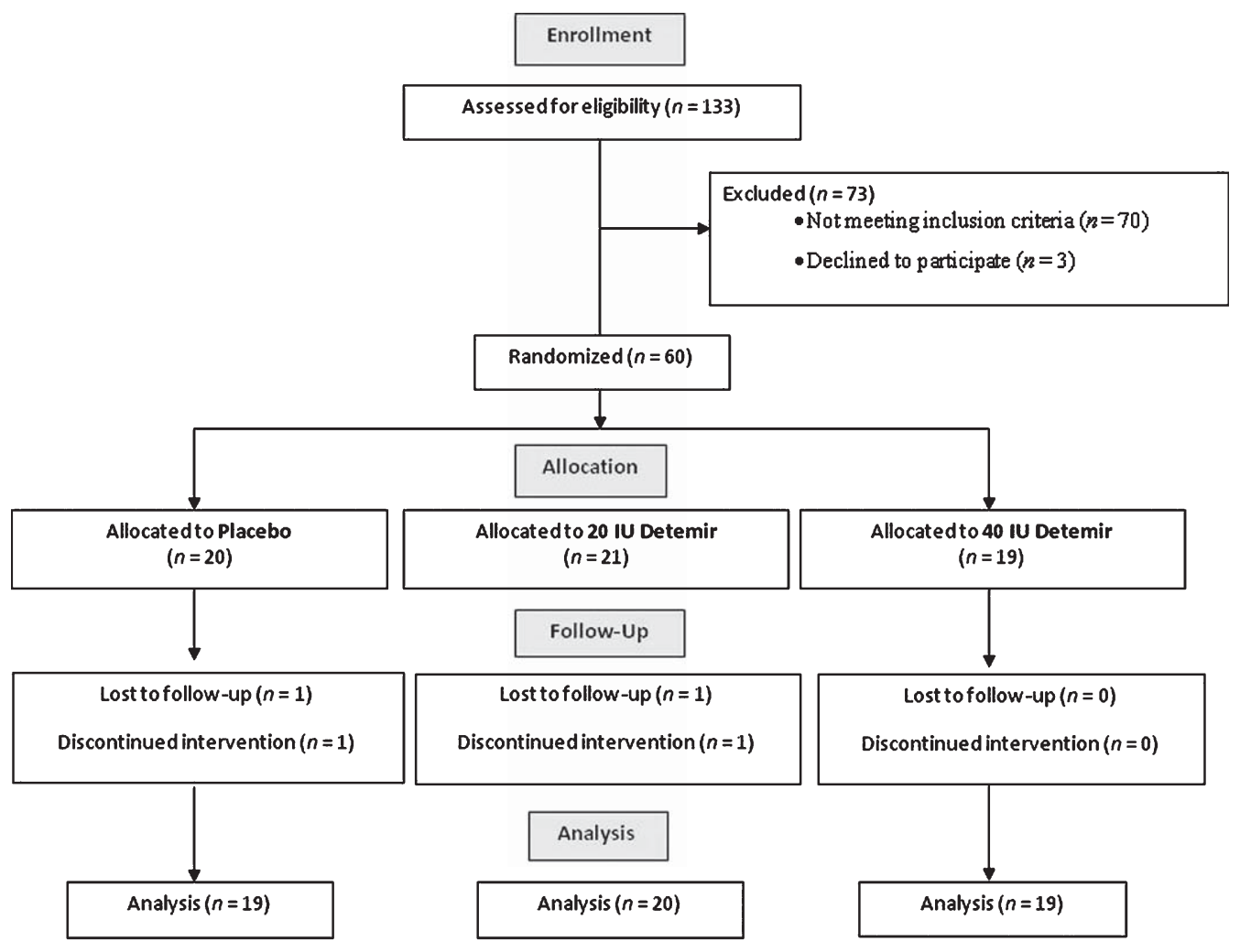

Fig. 1. Patient enrollment flowchart for the trial, which examines the effects of intranasal insulin detemir administration on cognition and function in adults with amnestic mild cognitive impairment or Alzheimer's disease.

immediate story recall, delayed story recall, immediate word list recall, and delayed word list recall. Immediate and delayed story recall [12] were determined after a story containing 44 informational bits was read a single time to participants, who were then asked to recall the story immediately and again after a 20-min delay. Immediate and delayed word list recall scores were derived from a 12-word Selective Reminding Word List task [33]. A higher score on the verbal memory composite indicates a better performance on these verbal memory measures. The four secondary outcome measures include tests of verbal working memory, visuospatial working memory, executive function, and caregiver-rated functional ability. Verbal working memory was measured by the Dot Counting N-back, in which participants were asked to count out loud the number of targets on consecutive computer displays. After $n$-number of displays, subjects recalled the number of targets presented on previous displays. Visuospatial working memory was assessed using the Benton Visual Retention Test (BVRT), Forms F and G, which is an object recognition memory paradigm [34]. For this task, subjects viewed a 2-D design and then identified this design included in an array containing three additional, highly similar distractors. Executive functioning was determined with a computer-administered version of Stroop ColorWord Interference task, a test of selective attention and response inhibition. In this task, color names were presented on a computer screen in concordant or discordant font colors (e.g., the word "red" was presented in either red or green font). For each of four alternating trial blocks, participants either read the word or named the font color as quickly as possible, and response latency (voice onset) and content were recorded. The reaction time variable was determined by taking the average of the response latency from each correct trial. Each trial was preceded by a displayed reminder of task instruction to minimize memory load. Finally, caregiver-rated functional ability was measured by the Dementia Severity Rating Scale (DSRS) [35], in which the study partner rated the change in the participant's cognitive, social, and functional status over a specified period of time, with higher scores indicating greater impairment.

Cognitive scores for each of the two detemir dose groups were compared to the placebo group using repeated measures analysis of covariance (ANCOVA). 
Age, diagnosis, MMSE score, gender, body mass index (BMI) and APOE- $\varepsilon 4$ allele status were statistically examined as covariates.

\section{Metabolic Measures}

Participants underwent oral glucose tolerance testing (OGTT) 1-2 days prior to cognitive baseline testing and study drug initiation and 1-2 days after cognitive testing on day 21 but prior to study drug discontinuation. Blood was collected from fasting participants and they then consumed a drink containing $75 \mathrm{~g}$ glucose. Blood was collected at $15,30,60,90$, and $120 \mathrm{~min}$ after beverage consumption. Samples were immediately placed on ice and spun at 2,200 rpm in a cold centrifuge for $15 \mathrm{~min}$, after which plasma, serum, lymphocytes, and red blood cells were aliquoted into separate storage tubes and flash frozen at $-70^{\circ} \mathrm{C}$. Blood glucose was measured via Accu-chek ${ }^{\circledR}$ glucose meters. Insulin resistance was calculated using the homeostatic model assessment of insulin resistance (HOMA-IR), a well-validated measure of insulin resistance calculated using fasting glucose and fasting insulin values obtained prior to administration of the OGTT beverage [36]. Another index of insulin resistance was derived from insulin and glucose levels during the OGTT: Insulin Area under the Curve (Insulin AUC) adjusted for glucose AUC was calculated using the trapezoidal rule in order to provide a secondary measure of insulin resistance that takes insulin response to a glucose challenge into account.

\section{Safety and Compliance}

Study partners supervised participants in the administration of intranasal treatment. Blood glucose levels were measured daily for the first week and then weekly thereafter; no episodes of hypoglycemia were observed. Compliance was monitored by quantifying unused medication and via self-report. Safety data were reviewed semiannually by a data safety monitoring board. Adverse event reporting followed standard guidelines. Fasting plasma glucose values obtained from each study visit are reported in Supplementary Table 1.

\section{Statistical Analyses}

Data were analyzed with SPSS version 18. For the intent-to-treat sample, primary and secondary cognitive and functional outcome scores were logtransformed to normalize distributions. To test the primary hypothesis that 21 days of treatment with detemir would improve cognition and daily function, the a priori analytic plan called for each of the insulin-treated groups to be compared with the placebo group. Outcomes scores were subjected to mixed-model repeated-measures analysis of covariance, including treatment group (placebo and $20 \mathrm{IU}$ of detemir; placebo and 40 IU of detemir) as the betweensubjects factor, and time (baseline and day 21) as the repeated factor, using the general linear models procedure, type III sums of squares. Age, diagnosis (MCI or $\mathrm{AD}$ ), gender, APOE- $\varepsilon 4$ carriage status (yes or no), BMI, baseline modified MMSE score, and years of education were also included as covariates. Nonsignificant covariates were dropped from the final model.

\section{RESULTS}

The three treatment groups did not differ at baseline on any outcome measure. There were also no group differences with respect to age, education, BMI, fasting insulin, fasting glucose, gender, APOE- $\varepsilon 4$ carriage status, or MMSE score. For ease of interpretation, the change in adjusted means from Time 1 (pre-treatment) to Time 2 (post-treatment) is graphed to illustrate significant results (Figs. 2-5). Non-log transformed baseline and post-treatment group means for all measures are included in Supplementary Table 2 and are presented by diagnosis in Supplementary Table 3.

\section{Primary Outcome}

No significant overall effects for the verbal memory composite were observed for the 20 or 40 IU dose comparisons. However, a significant treatment by time by APOE- $\varepsilon 4$ carriage interaction was observed for the 40 IU dose comparison $(p=0.03)$; interestingly, planned post hoc analyses revealed that APOE- $\varepsilon 4$ positive carriers taking $40 \mathrm{IU}$ intranasal detemir showed significant improvement in verbal memory $(p=0.02)$, whereas APOE- $\varepsilon 4$ negative participants taking 40 IU intranasal detemir showed a significant decline in verbal memory ( $p=0.02$ ) (see Fig. 2). No effects were observed for other covariates.

\section{Secondary Cognitive Outcomes}

Overall analyses revealed significant improvement for the $40 \mathrm{IU}$ group on both working memory tasks. For verbal working memory (Dot Counting N-back), a significant overall treatment group $\times$ time interaction was observed indicating improved verbal working memory 


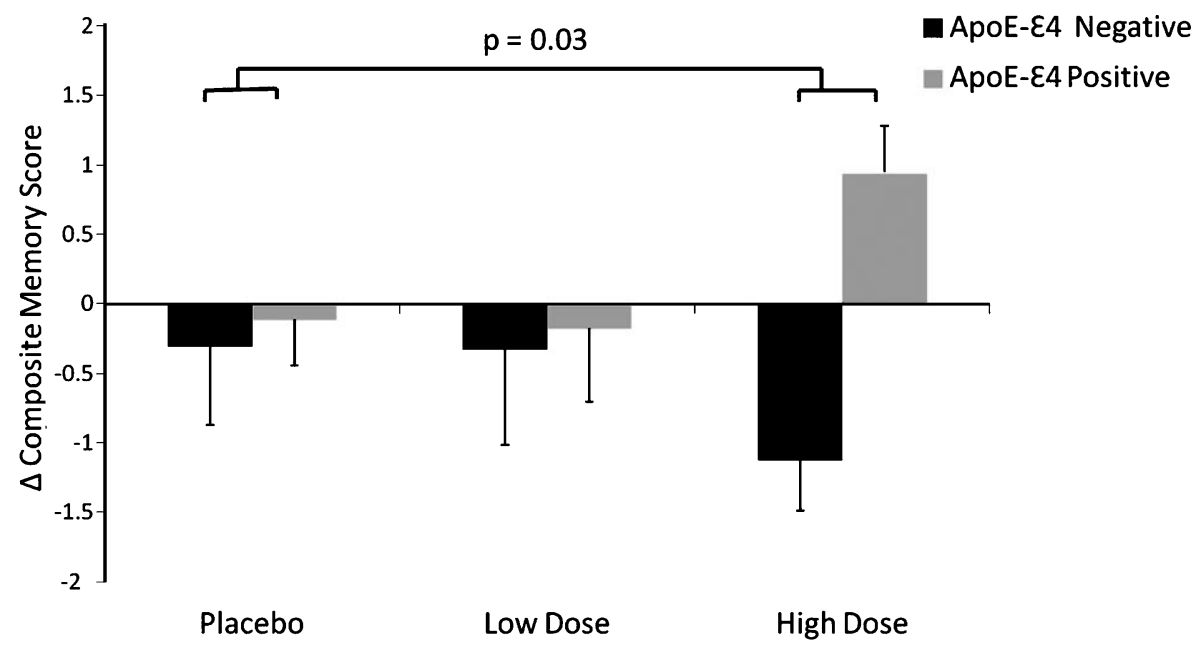

Fig. 2. Change in composite memory score from baseline to day 21, by treatment group and APOE- $\varepsilon 4$ carriage.

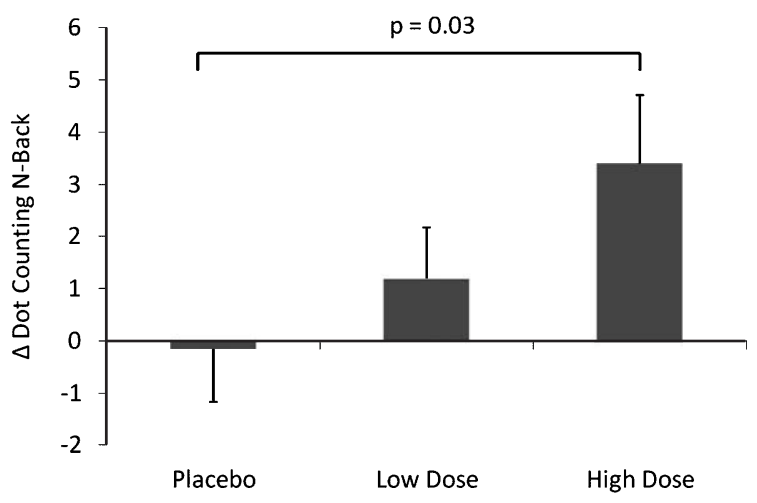

Fig. 3. Change in verbal working memory as measured by the Dot Counting N-Back Task from baseline to day 21, by treatment group.

in the $40 \mathrm{IU}$ group versus the placebo group $(p=0.03$; see Fig. 3). For visuospatial working memory (BVRT), a significant overall treatment group $\times$ time interaction was also observed ( $p=0.04$; see Fig. 4 ), indicating that subjects taking $40 \mathrm{IU}$ of intranasal detemir showed improved visuospatial working memory versus those in the placebo group. No significant interactions were observed with APOE status or other covariates, and no significant differences were noted for subjects who received the 20 IU dose versus placebo. For tests of executive function (Stroop) and daily functioning $(\mathrm{DSRS})$, there were no significant treatment $\times$ group effects for either dose comparison.

\section{Metabolic Outcomes}

As expected, higher baseline BMI was associated with higher baseline insulin resistance $(p<0.01)$.

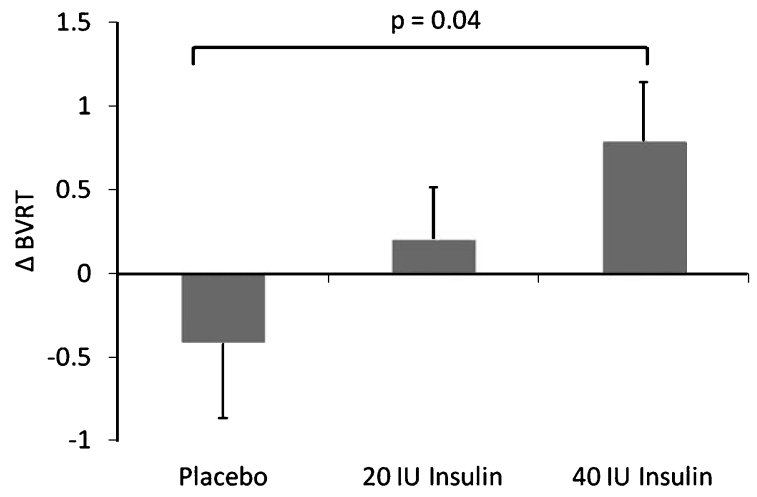

Fig. 4. Change in visuospatial working memory as measured by the BVRT from baseline to day 21 , by treatment group.

In addition, APOE- $\varepsilon 4$ carriage was associated with higher baseline Insulin AUC $(t=-2.05 ; p<0.05)$. There were no significant differences between APOE$\varepsilon 4$ carriers and non-carriers in the cholesterol profile (see Supplementary Table 4). For insulin resistance (HOMA-IR), the overall treatment $\times$ time effect was not significant. However, there was a treatment effect on insulin resistance with respect to APOE- $\varepsilon 4$ carriage status for the $40 \mathrm{IU}$ dose of detemir (treatment $\times$ time $\times$ APOE- $\varepsilon 4$ interaction: $p<0.01$ compared to the placebo group). For those with APOE- $\varepsilon 4$ negative status, the higher dose of intranasal insulin detemir was associated with an increase in insulin resistance across 21 days. For those with APOE- $\varepsilon 4$ positive status, taking intranasal detemir was associated with reduced insulin resistance across the 21 days (see Fig. 5). There were no significant treatment effects for Insulin AUC. 


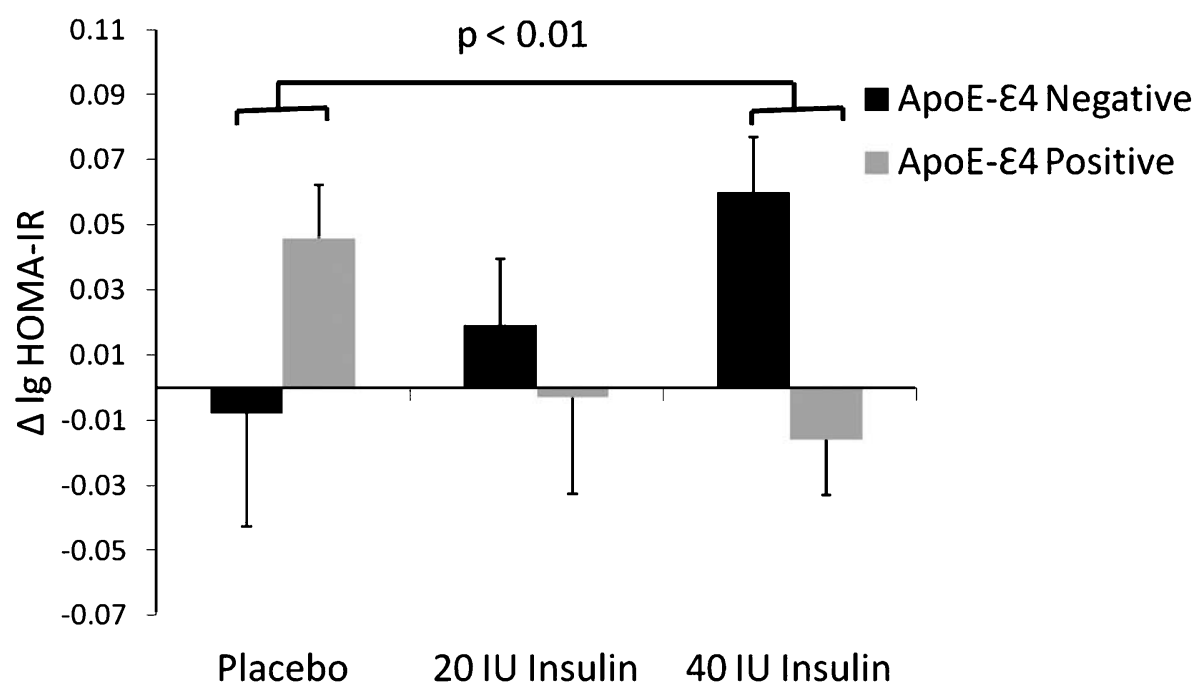

Fig. 5. Change in HOMA-IR from baseline to day 21, by treatment group and APOE- $\varepsilon 4$ carriage.

\section{Safety and Compliance}

No treatment-related severe adverse events occurred during the study, and most adverse events were minor, such as dizziness or mild rhinitis. There were no episodes of hypoglycemia. The adverse events are listed in Table 3. The total number of adverse events was lower for the $20 \mathrm{IU}$ detemir group compared with the placebo group $(p<0.05)$. Mean compliance (number of completed doses) was $98 \%$ and ranged from $89 \%$ to $100 \%$. Compliance did not differ across treatment groups.

\section{DISCUSSION}

Treatment with 40 IU intranasal detemir was associated with improved verbal memory for adults with MCI and AD who were APOE- $\varepsilon 4$ allele carriers, and improved visuospatial and verbal working memory for all participants. APOE- $\varepsilon 4$ allele carriers taking the higher dose of intranasal detemir also experienced improvement in peripheral insulin resistance across three weeks of treatment. Conversely, APOE$\varepsilon 4$ negative individuals treated with the higher dose of detemir experienced increased peripheral insulin resistance across three weeks of treatment. No effect was shown for executive functioning or caregiver-rated daily functioning with $40 \mathrm{IU}$ detemir, or for any of the cognitive outcomes after treatment with 20 IU detemir.

The current study marks the first time a long-acting insulin analogue has been administered intranasally to individuals with AD or amnestic MCI. This pilot study provides initial evidence that detemir can be safely administered to individuals with $\mathrm{AD}$ or MCI, and may enhance cognition for some groups. Of note, there were some key differences in the response to intranasal detemir compared with previous clinical trials that utilized regular insulin that support the possibility that insulin analogues may differ with respect to optimal therapeutic doses. Whereas a lower dose (20 IU daily) of regular insulin was effective for verbal memory in a previous study of individuals with AD or MCI, the $40 \mathrm{IU}$ dose of intranasal detemir was most consistently associated with positive outcomes in the current study. It is possible that the different pharmacodynamic profiles of insulin formulations explain this finding. While detemir has a longer half-life that results in greater cumulative exposure, regular insulin mimics postprandial release and reaches a higher peak, and thus may activate mechanisms underlying episodic memory enhancement at lower doses. Interestingly, rapid acting insulin aspart, which achieves the highest peak concentration after administration, reportedly has greater memory-enhancing effects than regular insulin [37]. Detemir also has a lower affinity for insulin receptors than does regular insulin, which may induce different dose requirements [38].

Our results provide additional evidence that APOE genotype may influence response to intranasal insulin treatment in adults with $\mathrm{MCI}$ and $\mathrm{AD}$. Interestingly, unlike previous studies with regular insulin, APOE- $\varepsilon 4$ positive carriers showed greater improvement in verbal episodic memory with 40 IU intranasal detemir. Although the mechanisms underlying this effect cannot be determined from the present study, detemir's greater lipophilicity and albumin-binding properties that are 
the basis of its protracted exposure profile may have played a role. Differences in amount or characteristics of albumin have been shown to modulate detemir's efficacy [39]. Albumin binding capacity and the albuminome are reportedly affected in various disease states such as cardiovascular and renal disease. Higher levels of post-translational glycation and nitration have reported in plasma and brain albumin in ADs patients that affect its ability to bind $A \beta[40,41]$. APOE- $\varepsilon 4$ carriers with AD have increased vulnerability to nitration [42], and thus may have a greater tendency for post-translational modifications of albumin, ultimately contributing to APOE-related differences in response to detemir [43]. These interesting possibilities require confirmation and further elucidation.

We also noted APOE-related differences in the effects of detemir on an index of insulin resistance, such that APOE- $\varepsilon 4$ participants in the 40 IU group had improved insulin resistance whereas participants without the APOE- $\varepsilon 4$ allele had worsened insulin resistance, changes which paralleled detemir effects on verbal episodic memory. Although peripherallyadministered detemir has been shown to improve glucose regulation in adults with diabetes, some investigators have suggested that prolonged insulin exposure may promote insulin resistance in vulnerable individuals. For example, detemir has been shown to worsen insulin resistance in adults with particular metabolic profiles that may associate with APOE genotype, such as non-alcoholic steatosis ("fatty liver") [44-46]. Although there were no differences between APOE- $\varepsilon 4$ carriers and non-carriers with respect to BMI or fasting glucose values, more in depth metabolic characteristics such as liver fat were not assessed.

It is also interesting that beneficial effects of 40 IU detemir on working memory were independent of APOE status. Working memory is preferentially mediated by the prefrontal and limbic systems [47], whereas verbal memory is associated with medial temporal/hippocampal circuits [48]. This pattern of results is consistent with previous clinical trials that show that different cognitive functions may have different dose response profiles [19], and underscore the importance of examining cognitive functions individually in addition to examining performance on global cognitive indicators.

An important goal of the present pilot study was to determine whether insulin detemir held sufficient promise as an $\mathrm{AD}$ treatment to support further investigation. Taken together, the benefits of detemir on memory and metabolic status provide a strong rationale for further examination of its therapeutic potential in
APOE- $\varepsilon 4$ carriers. In APOE- $\varepsilon 4$ non-carriers, a mixed picture was obtained, with improved working memory but worsened episodic memory and metabolic status. Additional study is needed to confirm this APOErelated pattern, potentially in a future Phase II study of moderate duration. If confirmed, this pattern would represent an important pharmacogenomic advance for AD therapeutics.

In conclusion, $\mathrm{AD}$ is a devastating illness, for which even small therapeutic gains have the potential to improve quality of life and significantly reduce the overall burden for patients, families, and society. Previous work has suggested that intranasal insulin may be a safe and effective treatment for the cognitive decline associated with AD. The current pilot study provides preliminary evidence that $40 \mathrm{IU}$ intranasal detemir may provide effective treatment for individuals diagnosed with MCI and AD dementia, and in particular for memory-impaired adults who are APOE- $\varepsilon 4$ carriers, a subgroup of patients notoriously resistant to therapeutic intervention [49]. Future longer-term studies are warranted to confirm this pattern and further examine the safety and efficacy for this promising treatment.

\section{ACKNOWLEDGMENTS}

Suzanne Craft and Amy Claxton had full access to all of the data in the study and take responsibility for the integrity of the data and the accuracy of the data analysis. This research was supported by National Institute of Aging grants P50 AG05136 (to Dr. Craft) and T32 AG000258 (to Dr. Claxton), and the Department of Veterans Affairs.

Authors' disclosures available online (http://www.j[alz.com/disclosures/view.php?id=2576]).

\section{SUPPLEMENTARY MATERIAL}

The supplementary material is available in the electronic version of this article: http://dx.doi.org/ 10.3233/JAD-141791.

\section{REFERENCES}

[1] Rivera EJ, Goldin A, Fulmer N, Tavares R, Wands JR, de la Monte SM (2005) Insulin and insulin-like growth factor expression and function deteriorate with progression of Alzheimer's disease: Link to brain reductions in acetylcholine. J Alzheimers Dis 8, 247-268.

[2] Gasparini L, Gouras GK, Wang R, Gross RS, Beal MF, Greengard P, Xu H (2001) Stimulation of beta-amyloid precursor protein trafficking by insulin reduces intraneuronal 
beta-amyloid and requires mitogen-activated protein kinase signaling. J Neurosci 21, 2561-2570.

[3] Craft S, Watson GS (2004) Insulin and neurodegenerative disease: Shared and specific mechanisms. Lancet Neurol 3, 169-178.

[4] Craft S, Peskind E, Schwartz MW, Schellenberg GD, Raskind M, Porte D, Jr. (1998) Cerebrospinal fluid and plasma insulin levels in Alzheimer's disease: Relationship to severity of dementia and apolipoprotein E genotype. Neurology 50, 164-168.

[5] Tomiyama $\mathrm{T}$ (2010) [Involvement of beta-amyloid in the etiology of Alzheimer's disease]. Brain Nerve 62, 691-699.

[6] Lee CC, Kuo YM, Huang CC, Hsu KS (2009) Insulin rescues amyloid beta-induced impairment of hippocampal long-term potentiation. Neurobiol Aging 30, 377-387.

[7] de la Monte SM, Wands JR (2008) Alzheimer's disease is type 3 diabetes-evidence reviewed. J Diabetes Sci Technol 2, 1101-1113.

[8] de la Monte SM (2012) Brain insulin resistance and deficiency as therapeutic targets in Alzheimer's disease. Curr Alzheimer Res 9, 35-66.

[9] Talbot K, Wang HY, Kazi H, Han LY, Bakshi KP, Stucky A, Fuino RL, Kawaguchi KR, Samoyedny AJ, Wilson RS, Arvanitakis Z, Schneider JA, Wolf BA, Bennett DA, Trojanowski JQ, Arnold SE (2012) Demonstrated brain insulin resistance in Alzheimer's disease patients is associated with IGF-1 resistance, IRS-1 dysregulation, and cognitive decline. J Clin Invest 122, 1316-1338.

[10] Bomfim TR, Forny-Germano L, Sathler LB, Brito-Moreira J, Houzel JC, Decker H, Silverman MA, Kazi H, Melo HM, McClean PL, Holscher C, Arnold SE, Talbot K, Klein WL, Munoz DP, Ferreira ST, De Felice FG (2012) An antidiabetes agent protects the mouse brain from defective insulin signaling caused by Alzheimer's disease- associated Abeta oligomers. J Clin Invest 122, 1339-1353.

[11] Reger MA, Watson GS, Green PS, Baker LD, Cholerton B, Fishel MA, Plymate SR, Cherrier MM, Schellenberg GD, Frey WH, 2nd, Craft S (2008) Intranasal insulin administration dose-dependently modulates verbal memory and plasma amyloid-beta in memory-impaired older adults. J Alzheimers Dis 13, 323-331.

[12] Reger MA, Watson GS, Green PS, Wilkinson CW, Baker LD, Cholerton B, Fishel MA, Plymate SR, Breitner JC, DeGroodt W, Mehta P, Craft S (2008) Intranasal insulin improves cognition and modulates beta-amyloid in early AD. Neurology 70 , 440-448.

[13] Craft S, Baker LD, Montine TJ, Minoshima S, Watson GS, Claxton A, Arbuckle M, Callaghan M, Tsai E, Plymate SR, Green PS, Leverenz J, Cross D, Gerton B (2012) Intranasal insulin therapy for Alzheimer disease and amnestic mild cognitive impairment: A pilot clinical trial. Arch Neurol. 69 , 29-38.

[14] Farrer LA, Cupples LA, Haines JL, Hyman B, Kukull WA, Mayeux R, Myers RH, Pericak-Vance MA, Risch N, van Duijn CM (1997) Effects of age, sex, and ethnicity on the association between apolipoprotein E genotype and Alzheimer disease. A meta-analysis. APOE and Alzheimer Disease Meta Analysis Consortium. JAMA 278, 1349-1356.

[15] Craft S, Asthana S, Schellenberg G, Cherrier M, Baker LD, Newcomer J, Plymate S, Latendresse S, Petrova A, Raskind M, Peskind E, Lofgreen C, Grimwood K (1999) Insulin metabolism in Alzheimer's disease differs according to apolipoprotein E genotype and gender. Neuroendocrinology 70, 146-152.
[16] Craft S, Asthana S, Schellenberg G, Baker L, Cherrier M, Boyt AA, Martins RN, Raskind M, Peskind E, Plymate S (2000) Insulin effects on glucose metabolism, memory, and plasma amyloid precursor protein in Alzheimer's disease differ according to apolipoprotein-E genotype. Ann N Y Acad Sci. 903, 222-228.

[17] Aisen PS, Berg JD, Craft S, Peskind ER, Sano M, Teri L, Mulnard RA, Thomas RG, Thal LJ (2003) Steroid-induced elevation of glucose in Alzheimer's disease: Relationship to gender, apolipoprotein E genotype and cognition. Psychoneuroendocrinology 28, 113-120.

[18] Craft S, Peskind E, Schwartz MW, Schellenberg GD, Raskind M, Porte D, Jr. (1998) Cerebrospinal fluid and plasma insulin levels in Alzheimer's disease: Relationship to severity of dementia and apolipoprotein E genotype. Neurology 50, 164168.

[19] Claxton A, Baker LD, Wilkinson CW, Trittschuh EH, Chapman D, Watson GS, Cholerton B, Plymate SR, Arbuckle M, Craft S (2013) Sex and ApoE genotype differences in treatment response to two doses of intranasal insulin in adults with mild cognitive impairment or Alzheimer's disease. J Alzheimers Dis 35, 789-797.

[20] Craft S, Asthana S, Cook DG, Baker LD, Cherrier M, Purganan K, Wait C, Petrova A, Latendresse S, Watson GS, Newcomer JW, Schellenberg GD, Krohn AJ (2003) Insulin dose-response effects on memory and plasma amyloid precursor protein in Alzheimer's disease: Interactions with apolipoprotein E genotype. Psychoneuroendocrinology $28,809-822$.

[21] Reiman EM, Chen K, Alexander GE, Caselli RJ, Bandy D, Osborne D, Saunders AM, Hardy J (2004) Functional brain abnormalities in young adults at genetic risk for late-onset Alzheimer's dementia. Proc Natl Acad Sci U S A 101, 284289.

[22] Kurtzhals P (2004) Engineering predictability and protraction in a basal insulin analogue: The pharmacology of insulin detemir. Int J Obes Relat Metab Disord 28, S23-28.

[23] De Leeuw I, Vague P, Selam JL, Skeie S, Lang H, Draeger E, Elte JW (2005) Insulin detemir used in basal-bolus therapy in people with type 1 diabetes is associated with a lower risk of nocturnal hypoglycaemia and less weight gain over 12 months in comparison to NPH insulin. Diabetes Obes Metab 7, 73-82.

[24] Shen DD, Artru AA, Adkison KK (2004) Principles and applicability of CSF sampling for the assessment of CNS drug delivery and pharmacodynamics. Adv Drug Deliv Rev 56, 1825-1857.

[25] Tschritter O, Hennige AM, Preissl H, Porubska K, Schafer SA, Lutzenberger W, Machicao F, Birbaumer N, Fritsche A, Haring HU (2007) Cerebrocortical beta activity in overweight humans responds to insulin detemir. PLoS One 2, e1196.

[26] Banks WA, Morley JE, Lynch JL, Lynch KM, Mooradian AD (2010) Insulin detemir is not transported across the bloodbrain barrier. Peptides 31, 2284-2288.

[27] Monami M, Marchionni N, Mannucci E (2009) Long-acting insulin analogues vs. NPH human insulin in type 1 diabetes. A meta-analysis. Diabetes Obes Metab 11, 372-378.

[28] Hennige AM, Sartorius T, Tschritter O, Preissl H, Fritsche A, Ruth P, Haring HU (2006) Tissue selectivity of insulin detemir action in vivo. Diabetologia 49, 1274-1282.

[29] Hallschmid M, Jauch-Chara K, Korn O, Molle M, Rasch B, Born J, Schultes B, Kern W (2010) Euglycemic infusion of insulin detemir compared with human insulin appears to increase direct current brain potential response and reduces food intake while inducing similar systemic effects. Diabetes 59, 1101-1107. 
[30] Hermansen K, Davies M, Derezinski T, Martinez Ravn G, Clauson P, Home P (2006) A 26-week, randomized, parallel, treat-to-target trial comparing insulin detemir with $\mathrm{NPH}$ insulin as add-on therapy to oral glucose-lowering drugs in insulin-naive people with type 2 diabetes. Diabetes Care 29, 1269-1274.

[31] Roberts RO, Geda YE, Knopman DS, Cha RH, Pankratz VS, Boeve BF, Ivnik RJ, Tangalos EG, Petersen RC, Rocca WA (2008) The Mayo Clinic Study of Aging: Design and sampling, participation, baseline measures and sample characteristics. Neuroepidemiology 30, 58-69.

[32] McKhann G, Drachman D, Folstein M, Katzman R, Price D, Stadlan EM (1984) Clinical diagnosis of Alzheimer's disease: Report of the NINCDS-ADRDA Work Group under the auspices of Department of Health and Human Services Task Force on Alzheimer's Disease. Neurology 34, 939-944.

[33] Coen RF, Kinsella A, Lambe R, Kenny M, Darragh A (2004) Creating equivalent word lists for the Buschke Selective Reminding Test. Hum Psychopharmacol Clin Exp 5, 47-51.

[34] Brickman AM, Stern Y, Small SA (2011) Hippocampal subregions differentially associate with standardized memory tests. Hippocampus 21, 923-928.

[35] Clark CM, Ewbank DC (1996) Performance of the dementia severity rating scale: A caregiver questionnaire for rating severity in Alzheimer disease. Alzheimer Dis Assoc Disord 10, 31-39.

[36] Matthews DR, Hosker JP, Rudenski AS, Naylor BA, Treacher DF, Turner RC (1985) Homeostasis model assessment: Insulin resistance and beta-cell function from fasting plasma glucose and insulin concentrations in man. Diabetologia 28, 412-419.

[37] Benedict C, Hallschmid M, Schmitz K, Schultes B, Ratter F, Fehm HL, Born J, Kern W (2007) Intranasal insulin improves memory in humans: Superiority of insulin aspart. Neuropsychopharmacology 32, 239-243.

[38] Sciacca L, Cassarino MF, Genua M, Pandini G, Le Moli R, Squatrito S, Vigneri R (2010) Insulin analogues differently activate insulin receptor isoforms and post-receptor signalling. Diabetologia 53, 1743-1753.

[39] Wada T, Azegami M, Sugiyama M, Tsuneki H, Sasaoka T (2008) Characteristics of signalling properties mediated by long-acting insulin analogue glargine and detemir in target cells of insulin. Diabetes Res Clin Pract 81, 269-277.
[40] Prajapati KD, Sharma SS, Roy N (2012) Hepatocyte nuclear factor-1alpha mediated upregulation of albumin expression in focal ischemic rat brain. Neurol Res 34, 25-31.

[41] Ramos-Fernandez E, Tajes M, Palomer E, Ill-Raga G, BoschMorato M, Guivernau B, Roman-Degano I, Eraso-Pichot A, Alcolea D, Fortea J, Nunez L, Paez A, Alameda F, Fernandez-Busquets X, Lleo A, Elosua R, Boada M, Valverde MA, Munoz FJ (2014) Posttranslational nitro-glycative modifications of albumin in Alzheimer's disease: Implications in cytotoxicity and amyloid-beta peptide aggregation. J Alzheimers Dis 40, 643-657.

[42] Colton CA, Needham LK, Brown C, Cook D, Rasheed K, Burke JR, Strittmatter WJ, Schmechel DE, Vitek MP (2004) APOE genotype-specific differences in human and mouse macrophage nitric oxide production. J Neuroimmunol 147, 62-67.

[43] Gundry RL, Fu Q, Jelinek CA, Van Eyk JE, Cotter RJ (2007) Investigation of an albumin-enriched fraction of human serum and its albuminome. Proteomics Clin Appl. 1, 73-88.

[44] Cao W, Ning J, Yang X, Liu Z (2011) Excess exposure to insulin is the primary cause of insulin resistance and its associated atherosclerosis. Curr Mol Pharmacol 4, 154-166.

[45] Cao W, Liu HY, Hong T, Liu Z (2010) Excess exposure to insulin may be the primary cause of insulin resistance. Am J Physiol Endocrinol Metab 298, E372.

[46] Mensenkamp AR, Havekes LM, Romijn JA, Kuipers F (2001) Hepatic steatosis and very low density lipoprotein secretion: The involvement of apolipoprotein E. J Hepatol 35, 816-822.

[47] Langner R, Sternkopf MA, Kellermann TS, Grefkes C, Kurth F, Schneider F, Zilles K, Eickhoff SB (2014) Translating working memory into action: Behavioral and neural evidence for using motor representations in encoding visuo-spatial sequences. Hum Brain Mapp 35, 3465-3484.

[48] Helkala EL, Laulumaa V, Soininen H, Riekkinen PJ (1988) Recall and recognition memory in patients with Alzheimer's and Parkinson's diseases. Ann Neurol 24, 214-217.

[49] Panza F, Frisardi V, Imbimbo BP, D'Onofrio G, Pietrarossa G, Seripa D, Pilotto A, Solfrizzi V (2010) Bapineuzumab: Anti-beta-amyloid monoclonal antibodies for the treatment of Alzheimer's disease. Immunotherapy 2, 767-782. 